\title{
Substance Change in Pro-Apoptosis and Pre- Apoptosis in Malignant Transformation of Cell Clusters in Carcinogenesis
}

\author{
Lawrence M Agius* \\ Department of Pathology, University of malta medical school, Europe
}

Submission: March 20, 2018; Published: April 06, 2018

"Correspondence Address: Lawrence M Agius, Mater dei hospital, TAL-QROQQ, University of Malta medical school, MSIDA, Malta Europe, Tel: 356-21451752; Email: lawrence.agius@um.edu.mt

\begin{abstract}
Dynamics of pro-apoptosis and of pre-apoptosis are significant potential states of activity that well-characterize the malignant transformation event in cell clustering and progression. The nature of attribute determination allow a permissive pre-determination that strictly contributes to a realization in terms of the established formulas for progression of cascade events per se and as further outlined by systems of carcinogenesis and spread. The parameters for such characterization are idealized definitions for significant predispositions that profile formulates the dimensions for cooperative events as strictly interface formulas in their own right and as described pathway consequence as clearly defined by the malignant transformation event in carcinogenesis.
\end{abstract}

\section{Introduction}

Pro-apoptosis refers to a series of enhanced apoptotic predispositions inherently arising from interface susceptibility consequences between extrinsic and intrinsic apoptosis within the living cell in a manner that predominantly favors dysfunction of ligand-binding of initiator mechanisms. In such terms, the evolutionary dynamics of pro-apoptosis is associated with resistance to the onset and maintenance of cell proliferative states. MicroRNAs are evidently powerful regulators of maintained cellular homeostasis and immune evasion [1].

The degrees of progressive rates of susceptibility creation of apoptosis events includes dynamics borne out by a series of death domains in a manner specific to the incremental progression of susceptibility to apoptotic activity in terms of the conformational binding of such aggregate phenomena as apoptosome and DISC dynamics. Apoptosis-stimulating protein of p53 forms an interregulatory loop with Early Growth Response 1 and promotes apoptosis via inhibiting cyto-protective autophagy, independent of the well-documented p53-dependent mechanisms [2]. Complex creation is a specific conformational consequence of such interface dynamics that include the prevalent molecular forms of such entities as decoy death receptivity. RBM5 is a RNAbinding motif protein and is reported as a tumor suppressor, inhibiting Wnt/catenin signaling and inducing apoptosis in gliomas [3].

\section{Pro-Apoptosis}

The inclusive proliferative response of pro-apoptosis is a considered re-interpretation of events as induced by NuclearFactor kappaB motivated dynamics. Bid exerts pro-apoptosis effects, but also promotes cell proliferation, maintains S phase checkpoint and facilitates inflammasome activation [4]. Such phenomenon is a semblance formula for the further derived and substantial re-formulation as strictly characterized by systems of decoy nature. The extrinsic phenomenon of receptor-ligand binding is paramount acquisition of the extra-cellular dynamics that progress specifically as transduction pathways of activation of primary and sequentially evoked binding dynamics of caspase nature and origin. Putative cancer stem cells present the transcriptomic profile of multipotent cells [5]. The significance of a homo-trimeric identity to initiator caspase activities is further projected in terms of inter-active formulas for further progression in the form of specific executioner caspases. Rapamycin specifically affects Bax and survivin signaling pathways in activated apoptosis [6].

The definitive outcome of pro-apoptosis is hence a disordered regulatory dimension that correlates also in terms of interface molecular events of binding and disjunction between initiator and executioner caspases. 
It is further to such considerations that dynamic forms of receptor binding are primarily outcome phenomena in internalized turnover of systems of cooperative dynamics, as further evidenced by systems of re-formulated objects of progressive enhancement or inhibition of the interface interactivities. The epigenetic regulation of TSSC3, a proapoptosis gene, provides valuable insights in osteosarcoma therapy with loss of expression of the imprinted gene [7].

\section{Resistance}

The resistance profiles of various tumor isotypes reflect the summation phenomena of pro-apoptotic states in relative comparison and contrast to the onset dynamics of programmed cell death pathways. Ericifolin, an antitumor agent from allspice, silences androgen receptors in prostate cancer and shows anti-proliferative, pro-apoptotic and anti-Androgen receptor activities [8]. In such manner, the progressive nature and systems of enhanced programmed cell death clearly indicate potential attributes of ligand-receptor binding in terms of transduction pathways. The onset systems of progression are themselves maintenance mechanisms in formulated dimension that redefine the persistence of pathway cascades and that also allow further understanding in terms of the acquisition to apoptosis susceptibilities.

Flavonoids possess anti-angiogenetic, anti-metastasis and pro-apoptosis properties and decreases nuclear levels and binding ability of nuclear factor-kappaB and activator protein-1 [9].

\section{Cleavage}

Cleavage phenomena are derived attributes to the onset determination of cascade events as integral to the profile dynamics of the system identities as predominantly determined by executioner caspase activity. A novel framework that distinguishes distinct patterns of gene co expression networks and inflammation-based modules shows advantages of an integrative and comparative network analysis over the differential expression-based approach and virus-host interactome-based approach [10]. The realization of system profiles indicates a susceptibility state that is deformed by cellular malignant change and that is further labeled in terms of the onset dynamics of such maintenance programs as programmed cell death pathways. Reexpressed CXCL14 is a common target in epigenetic silencing in lung cancer and provokes tumor necrosis [11].

\section{System Profiles}

The realization of system profiles is it clears recognition of interface development of molecular cascades that permit and further allow the emergence of interactions between the extrinsic and intrinsic apoptosis phenomena. It is further evident that substantial acquisition to molecular dynamics clarifies the attributes of pro-survival of cells as negative or positive acquisition of the pro-apoptosis of individual cells in terms of clustering phenomenon of molecular species.
The proposed increments in molecular binding allow for predisposed enhancement as further borne out by the profile outlines as determined by systems of created potentiality. Protocadherin 10 is a gastric tutor suppressor and its methylation at an early set of carcinogenesis is an independent prognostic factor [12]. It is further to such molecular binding to receptors and decoy receptors that a concept of molecular dynamics is a reflected dimension of turnover in terms that predispose to conformational modification and to post-translational change in the binding of interface modalities. Substantial attributes of dysfunction are further accentuated within predisposed susceptibilities to a state of enhanced apoptotic susceptibility, as terms that additionally require the outline emergence for further progressive cascades of interactivity, A radiation-induced acute apoptosis involving TP53 and BAX precede delayed apoptosis and malignant transformation of CGL1 human hybrid cells derived from (HeLa X fibroblast)[13].

\section{Deliverance}

The deliverance components that term-define apoptosis as programmed cell death allow for the system determination of cell death as conceptually re-defined by formulas of interactivity. The profile contributions of susceptibility ratios in molecular terms allow for the emergence of profile phenomena that strictly characterize dimensions of progression as systems of maintenance of the cellular malignant transformation event. Flubendazole suppresses glioma cell division by G2/M cell cycle arrest and provokes pro-apoptosis [14].

Attribute determination is therefore significant as profile potentiality in its own right, as further determined in set terms of onset and progression of the cascade events of serial caspase cleavage events. It is beyond the characterization of significant formulation that systems of recognition are systems of progression in receptor-binding events. The inhibition of autophagy may constitute a novel mode to overcome tolerance of glioblastoma to anti-angiogenic agents [15]. The clear predetermination of susceptibility traits allow for the significant emergence of the pre-apoptosis as an integral event in response to the malignant transformation event per se, as further dictated by formulas of potential execution of pathway cascades. Selenium induces apoptosis and oxidative stress in oral carcinomatous cells when provided in high concentrations [16].

\section{Onset}

The significant correlates of onset dynamics of the apoptosis cascades as terms of reference to maintenance formulas in programmed cell death allow for a realized dimension of cooperative phenomena that belie the significant predisposition to malignant change. In such terms, cooperative potentiation of the malignant transformation event predispose cells to clustered reformulations in terms of identity ratios as further projected by systems of susceptibility and of maintained profiles of such susceptibility. The realization of malignant transformation is further corroborated by the onset outcome of pre-determined 


\section{Cancer Therapy \& Oncology International Journal}

formulas for further change as well-determined by the outline dynamics for potential further change in contribution acquisition.

\section{Conclusion}

Interface collaboration is a strictly acquired system profile that characterizes and re-characterizes the onset dynamics of pro-apoptosis in terms of the subsequent performance of programmed cell death. It is further to be realized the dynamic interplay of system profiles as determined by the inclusion of cell surface membrane receptivity, on the one hand, and the clustering dynamics of binding to the mitochondrial outer membrane of caspases. It is significant to consider the profile schemes for pre-determination as realized interface consequences of the extrinsic and intrinsic apoptosis in defined formulas for contributed maintenance of pro-apoptosis throughout the course progression of the programmed cell death phenomenon.

\section{References}

1. Zuo L, Yue W, Du S, Xin S, Zhang J, et al. (2017) An update: Epstein-Barr virus and immune evasion via microRNA regulation. Virol Sin 32(3): 175-187.

2. Zhao K, Yu M, Zhu Y, Liu D, Wu Q et al. (2017) EGR-1/ASPP1 interregulatory loop promotes apoptosis by inhibiting cyto-protective autophagy. Cell Death Dis 8(6): e2869.

3. Jiang Y, Sheng H, Meng L, Tue H, Li B, et al. (2017) RBM5 inhibits tumorigenesis of gliomas through inhibition of Wnt/catenin signaling and induction of apoptosis. World J Surg Oncol 15(1): 9.

4. Yu C, Yan S, Khambu B, Chen X, Dong Z, et al. (2015) Gene expression analysis indicates divergent mechanisms in DEN-induced carcinogenesis in wild-type and Bid-Deficient livers. PLoS One 11(5): e0155211.

5. Zakaria N, Yusoff NM, Zakaria Z, Lim MN, Bahanuddin PJ, et al. (2016) Human non-small cell lung cancer expresses putative cancer stem cell markers and exhibits the transcriptomic profile of multipotent cells. BMC Cancer 15: 84.

6. Al-Astani Tengku Din TA, Shamsuddin SH, Idris FM, Griffin Wan Mansor WN, Abdul Jatal M, et al. (2014) Rapamycin and PF4 induce apoptosis by up regulating Bax and down-regulating survivin in MNU-induced breast cancer. Asian Pac J Cancer Prev 15(9): 3939- 3944.

7. Li Y, Huang Y, LvY, Meng G, Gut QN, Qiao Nan Guo (2014) Epigenetic regulation of the pro-apoptosis gene TSSC3 in human osteosarcoma cells. Biomed Pharmacother 68(1): 45-50.

8. Shamaladevi N, Lyn DA, Shaaban KA, Zhang L, Villate S, et al. (2013) Ericifolin: a novel antitumor compound from allspice that silences androgen receptor in prostate cancer. Carcinogenesis 34(8): 18221832.

9. Li F, Li C, Zhang H, Lu Z, Li Z, et al. (2012) Vl-14, a novel flavonoid derivative, inhibits migration and invasion of human breast cancer cells. Toxic Appl Pharmacy 261(2): 217-226.

10. He D, Liu ZP, Honda M, Kaneko S, Chen L (2012) Coexpression network analysis in chronic hepatitis $\mathrm{B}$ and $\mathrm{C}$ hepatic lesions reveals distinct patterns of disease progression to hepatocellular carcinoma. J Mol Cell Biol 4(3): 140-152.

11. Tessema M, Klinge DM, Yingling CM, Do K, Van Neste L, et al. (2010) Reexpression of CXCL14, a common target for epigenetic silencing in lung cancer, induces tutor necrosis. Oncogene 29(37): 5159-5170.

12. Yu J, Cheng YY, Tao Q, Cheung KF, Lam CN, et al. (2009) Methylation of protocadherin 10, a novel tumor suppressor, is associated with poor prognosis in patients with gastric cancer. Gastroenterology 136(2): 640-651.

13. Mendonca MS, Mayhugh BM, McDowell B, Chin-Sinex H, Smith ML, et al. (2005) A radiation-induced acute apoptosis involving TP53 and BAX precedes the delayed apoptosis and neoplastic transformation of CGL1 human hybrid cells. Radiat Res 163(6): 614-622.

14. Zhou X, Liu J, Zhang J, Wei Y, Li H (2018) Flubendazole inhibits glioma proliferation by G2/M cell cycle arrest and pro-apoptosis. Cell Death Discov 4: 18.

15. Huang H, Song J, Liu Z, Pan L, Xu G (2018) Autophagy activation promotes bevacizumab resistance in glioblastoma by suppressing Akt/ mTOR signaling pathway. Oncol Lett 15(2): 1487-1494.

16. Qiao B, He B, Cai J, King-Yin Lam A, He W (2017) Induction of oxidative stress and cell apoptosis by selenium: the cure against oral carcinoma. Oncotarget 8(69): 113614-113621.

Your next submission with Juniper Publishers will reach you the below assets

- Quality Editorial service

- Swift Peer Review

- Reprints availability

- E-prints Service

- Manuscript Podcast for convenient understanding

- Global attainment for your research

- Manuscript accessibility in different formats ( Pdf, E-pub, Full Text, Audio)

- Unceasing customer service

Track the below URL for one-step submission https://juniperpublishers.com/online-submission.php 